\title{
UNCHARTED WATERS: AN EXEGETICAL EXPLORATION OF GHANA'S REGULATORY FRAMEWORK IN RELATION TO CRYPTOCURRENCY
}

\author{
Gideon T Gabor \\ ABSTRACT
}

T he historical evolution of money has taken many forms such as precious metals, cowries, banknotes and coins, with the latest stage of this evolving continuum being digital currency. This evolution has been characterised by the gradual movement to a more cashlite aware society. The transition is being facilitated by constant improvement in financial technologies and services. Ghana is no exception to this development. The emergence of credit and debit cards as well as mobile banking is digitising Ghana's currency whilst extending financial services across the country. Mobile money payment facilitated by the telecommunication companies in partnership with the traditional banks is also fast-tracking Ghana's progress towards a cashlite economy. In 2009, a new cash-like electronic instrument known as Bitcoin emerged. Bitcoin is touted as offering anonymous, fast and irreversible peer to peer transactions, across borders with little or no transactional cost. The introduction of Bitcoin has birthed the cryptocurrency era. The anonymous nature of cryptocurrency transactions is an attraction to criminals and terrorist organisations who use cryptocurrency to facilitate unlawful activities. Despite the enormous financial, social and economic, and even political risk in cryptocurrency use, this financial instrument is largely unregulated in most countries. The potential use of cryptocurrency for purposes outside the law justifies the need for regulation to make cryptocurrency use less attractive for unlawful activities. This article draws on the legal approaches to regulating cryptocurrency by adopting a desk research to theoretically examine and interpret primary and secondary sources of data in order to determine the possible existence of an existing Ghanaian legal framework regulating cryptocurrency in Ghana.

Keywords: Ghana; Regulations; Digital Currencies; Cryptocurrency.

1 Q.C.L. candidate (Ghana School of Law); LL. B (Ghana Institute of Management and Public Administration). I am grateful to Godwin Dzah of the Faculty of Law, GIMPA for his mentorship and guidance. A modified version of this article was previously submitted and has been published in the 2020 Ghana School of Law Student Journal. All errors remain mine. 


\section{Introduction}

Cryptocurrency is a global phenomenon, demonstrated to offer real and potential benefits such as providing financial services to the unbanked and underbanked, through fast and cheap cross border transactions. ${ }^{2}$ Notwithstanding, cryptocurrency is also uniquely suited to facilitate unlawful activities. ${ }^{3}$ The illicit use of cryptocurrency has been identified as negligible when juxtaposed with the illicit use of well-established payment methods such as paper money. ${ }^{4}$ However cryptocurrency's potential of 'disruptively innovating'5 the existing financial industry presents the need for regulation to limit the attractiveness of cryptocurrency for illicit use.

In Ghana, public interest in relation to cryptocurrency has been on the rise. ${ }^{6}$ This interest was further evidenced when some Ghanaian business leaders urged upon the Bank of Ghana (BoG) to diversify its investments by investing in the cryptocurrency, Bitcoin. ${ }^{7}$ However, some banks in Ghana have expressed strong resistance to the adoption of cryptocurrency in Ghana. ${ }^{8}$ The banks cited the narrative of an absent regulatory framework, regulating cryptocurrency in Ghana as the basis for their scepticism. ${ }^{9}$

It is inevitable that disputes with all the concomitant legal suits may soon arise in relation

2 Flamur Bunjaku and others, 'Cryptocurrencies-Advantages and Disadvantages' (2017) 2(1) Journal of Economics 31, 37-38.

3 Marian demonstrates how the combination of anonymity, and the decentralisation of financial dealings make cryptocurrency uniquely suited for criminal activity: Omri Marian, 'A Conceptual Framework for the Regulation of Cryptocurrency' (2017) 82(53) The University of Chicago Law Review 53, 56-57.

4 David Carlisle, 'Virtual Currencies and Financial Crime Challenges and Opportunities' (2017) Royal United Services Institute for Defence and Security Studies, 5.

5 Christensen attributed with the coining of this term, describes the term as a process by which an innovation transforms an existing market or sector, initially taking root in simple applications at the bottom of a market, and then relentlessly moves upward and completely redefining the industry as it creates entirely new markets with different value networks: Clayton Christensen, The Innovator's Dilemma: When New Technologies Cause Great Firms to Fail (Cambridge 1997); Jonathan Lim, 'A Facilitative Model for Crypto-Currency Regulation in Singapore' (2014) Centre for Banking and Finance Law, 1.

6 Google search interest on cryptocurrency such as Bitcoin has heightened in the past 12 months in Ghana with Ghana placing second in Africa after South Africa.; Google Trends <https://trends.google. com/trends/explore?q=bitcoin> accessed 2 January, 2019.

7 Joseph Appiah-Dolphyne, 'Invest 1\% of Ghana's reserves in Bitcoin Ndoum to BOG' (Joyonline, 2 January 2018) <https://www.myjoyonline.com/business/2018/January-2nd/invest-1-of-ghanas-reservesin-bitcoin-ndoum-to-bog.php> accessed 15 January, 2019.

8 Pius Eduku, 'Banks resist crypto currency use in Ghana' (Citifmonline, 16 March 2018) <http:// citifmonline.com/2018/03/16/banks-resist-crypto-currency-use-ghana/> accessed 24 January 2019.

9 ibid. 
to cryptocurrency in Ghana. ${ }^{10}$ This article, drawing on the narrative of an absent regulatory framework regulating cryptocurrency in Ghana, engages with the literature - primary and secondary - on cryptocurrency to analyses cryptocurrency under a Ghanaian legal scope.

Aside this first section which is the introduction, this article further proceeds in four sections. The second section examines digital currencies, specifically cryptocurrency and its unique advantage in aiding illicit activities. The third section considers the cryptocurrency regulatory discourse, analysing in detail, the literature in relation to the regulation of cryptocurrency. Section four builds on the analysis in section three to further explore the existence of cryptocurrency regulation in Ghana. The fifth and final section concludes the article.

\section{Digital Currencies}

The advent of the internet has spawned new financial technologies and services that are transforming the global financial sector. ${ }^{11}$ This is heralding an era of cashlite aware societies characterised by the increase in use of e-money. ${ }^{12} \mathrm{E}$-money, as used in this context, is a digital representation of fiat currency used to electronically transfer value denominated in fiat currency. ${ }^{13}$ The electronic nature of e-money transfers means, money can be transferred effortlessly across borders. The increase in use of e-money is rapidly transforming the transfer of money, from the physical exchange of banknotes and coins to the debit and credit of accounts of transacting parties. At the core, the transfer of e-money is simply a transfer of a digital asset ${ }^{14}$ similar to the transfer of a text

10 An alleged cryptocurrency Ponzi scheme alleged to be accepting deposits and engaging in investment schemes are being investigated by the EOCO. The legal status of cryptocurrency made be considered if criminal procedures are initiated: Suleiman Mustapha, 'EOCO grills crypto-currency directors over GH $\phi 135$ m customers' cash' (Graphic Online, 29 November 2018) <https://www.graphic.com.gh/news/ general-news/ghana-news-eoco-grills-crypto-currency-directors-over-gh-135m-customers-cash.html> accessed 13 March 2019.

11 Asli Demirgüç-Kunt and others, The Global Findex Database Measuring Financial Inclusion and the Fintech Revolution (World Bank Publications 2017).

12 Capgemini and BNP Paribas, 'World Payment Report 2017' < https://www.worldpaymentsreport. com> accessed 13 March 2019.

13 Financial Action Task Force, 'Virtual Currencies: Key Definitions and Potential AML/CFT Risk (2014) Financial Action Task Force Report, 4 [hereinafter referred to as FATF Definition].

14 Sherry defines a digital asset as anything stored in a digital file: Kristina Sherry, 'What Happens to Our Facebook Accounts When We Die? Probate Versus Policy and the Fate of Social-Media Assets Postmortem' (2012) 40(1) Pepperdine Law Review 185, 194. 
or music file; thus it can be duplicated and spent multiple times. ${ }^{15} \mathrm{~A}$ trusted third party institution such as a bank or credit card entity process e-money transactions at a cost, potentially eliminating the double spending problem. ${ }^{16}$ The trusted third party institution is usually represented by a centralised bank which controls the issuance and withdrawal of e-money; and validates and verifies e-money transactions. ${ }^{17}$ E-money transactions are thus subjected to the rules and bureaucracies of such trusted third party institutions. ${ }^{18}$

\section{Advent of Cryptocurrency}

In November 2008, a seminal white paper, authored under the pen name of Satoshi Nakamoto, described a peer to peer electronic cash system known as Bitcoin. ${ }^{19}$ In January 2009, the first Bitcoin was issued. Bitcoin's issuance and transaction is protected by cryptography ${ }^{20}$ thus introducing the world's first cryptocurrency. The success of Bitcoin spurred the proliferation of a plethora of alternative cryptocurrency (altcoins) including Litecoin, Ethereum and Ripple, which implement similar yet modified cryptographic features of Bitcoin. ${ }^{21}$ The current number of distinct cryptocurrency in circulation is pegged at 2110 , with a significantly combined market capitalisation of about $\$ 364$ billion. ${ }^{22}$

Cryptocurrency, like e-money, is electronic in nature, thus connects transacting parties across borders. Cryptocurrency distinguishing features includes the nature of

15 This term is described as the double spending problem: Aleksander Berentsen and Fabian Schär, 'A Short Introduction to the World of Cryptocurrencies' (2018) 100(1) Federal Reserve Bank of St. Louis Review, 2.

16 ibid.

17 FATF Definition (n 13) 7.

18 The bureaucracies include restricting transactions during business hours, holidays; limiting transactions and issuing barriers to entry; Satoshi recognises the inherent weakness of such trusted third party institutions and posits the idea of an electronic cash system eliminating such institutions: Satoshi Nakamoto, 'Bitcoin: A Peer-to-Peer Electronic Cash System' (2008) <https://bitcoin.org/bitcoin.pdf> access 24 January 2019.

19 ibid.

20 Cryptography is use of mathematics to create codes and ciphers that can be used to conceal information:

Secretary of the Department of Homeland Security, Risks and Threats of Cryptocurrencies (Homeland Security Studies and Analysis Institute 2014) xii [hereinafter referred to as Homeland Security]; Edward Murphy, 'Bitcoin: Questions, Answers, and Analysis of Legal Issues' (2015) Congressional Research Service, 1.

21 Primavera De Filippi, 'Bitcoin: A Regulatory Nightmare to a Libertarian Dream' (2014) 3(2) Internet Policy Review 43, 46.

22 The market capitalisation of a particular cryptocurrency is the value of the total available number units of a particular cryptocurrency in circulation; CoinMarketCap <https://coinmarketcap.com> accessed 20 October 2020. 
cryptocurrency and the way it solves the double spending problem. Cryptocurrency unlike e-money is not a substitute of fiat currency but a form of currency on its own. In mimicking fiat currency units, a cryptocurrency is represented by a distinct unit of account such as BTC for Bitcoin and Ether for Ethereum. It is important to point at this early stage that a unit of cryptocurrency is intrinsically worth nothing. Cryptocurrency derive their value from the belief and confidence that they might be exchanged for goods or services; or converted to an amount of fiat currency. The belief in a subjective value has resulted in the volatility of the value of cryptocurrency. ${ }^{23}$

Beyond volatility, cryptocurrency as a digital asset, is potential susceptible to the double spending problem. ${ }^{24}$ It solves the double spending problem by relying on a public ledger system and the group consensus of network participants to verify and validate transactions. This public ledger system, known as the Blockchain, ${ }^{25}$ is a ledger recording ownership and transfers of units of cryptocurrency, with each record timestamped and referencing the earlier transaction. The Blockchain relies on cryptography to secure the ledger. This ledger system is characterised as public, as it distributes a copy of the ledger to all network participants connected to that particular Blockchain. The network participants can then track and trace every transaction on the Blockchain and ensure through group consensus that a user is not 'double spending' the same unit of cryptocurrency. A verified and validated transaction is then recorded on the Blockchain. A recorded transaction cannot be reversed. Transactions are also transparent and accessible to every person connected to the Blockchain. Attempts at altering an individual copy of the Blockchain would conflict with the copies of the Blockchain of other users, consequently ensuring the ledger is secure from hacks and unverified changes. The decentralised nature of cryptocurrency has thus eliminated the need for the centralised trusted third party institution with its fees, rules and failures. ${ }^{26}$ Cryptocurrency is marked by peer to peer transactions and lower transaction costs, thus users are at liberty to send any units of cryptocurrency anywhere in the world irrespective of public policy and international 23 On $17^{\text {th }}$ December, 2017 the value of the cryptocurrency, Bitcoin, reached an all-time high of about $\$ 19,783$ for one BTC. This seems to have a ripple effect on most cryptocurrency which traded at all-time highs during that period: David Morris, 'Bitcoin Hits a New Record High, But Stops Short of $\$ 20,000$ ' (Fortune, 17 December 2017) <http://fortune.com/2017/12/17/bitcoin-record-high-short-of-20000/> accessed 21 January 2019.

24 Andres Guadamuz and Chris Marsden, 'Blockchains and Bitcoin: Regulatory Responses to Cryptocurrencies' (2015) 20(12) First Monday, 12

25 The Blockchain has been described as 'one of the most fundamental inventions in the history of computer science and the invention at the heart of the fourth industrial revolution': Kobina Hughes, 'Blockchain, The Greater Good, and Human and Civil Rights' (2017) 48(5) Metaphilosophy, 1.

$26 \operatorname{Lim}($ (n) 5. 
economic sanctions. ${ }^{27}$ As Bill Gates stated, '[B]itcoin is exciting because it shows how cheap transfer of fund from one place to another can be'. ${ }^{28}$

Furthermore, the Blockchain as already stated is transparent, and at the same time, maintains a level of privacy of owners of units of cryptocurrency. This is done by representing the real identities of users with a pseudonym represented by a string of alphanumerical characters. Hence no personal identifiable information of the user is stored on the Blockchain. Cryptocurrency transactions are anonymous and users are thus cloaked in pseudonymity. It is this perceived anonymity of cryptocurrency transactions that is so often exploit by cybercriminals and terrorist organisations that use cryptocurrency as a medium of payment for illicit goods and services, to facilitate money laundering and promote terrorist activities. ${ }^{29}$

\section{Illicit Use of Cryptocurrency}

Europol notes that, '[F]or almost all types of organised crime, criminals are deploying and adapting to technology with ever greater skill and to ever greater effect, with organised crime moving increasingly to the dark web'. ${ }^{30}$ Cryptocurrency anonymous, rapid, borderless, cheap and irreversible transfers have thus been beneficial to bad actors such as cybercriminals, terrorist organisations and dealers in illicit narcotics. Carlisle identifies the narrative that, cryptocurrency enables the flourishing of new types of crime such as dark web activity and increased ransomware attacks. ${ }^{31}$

The most well-known criminal use of cryptocurrency is as a payment method for illicit goods and services such as drugs, child pornography, counterfeited documents, as well as, weapons and ammunition offered on hidden online market sites hosted on the dark

27 Reuters, 'Venezuela cryptocurrency to draw investment from Turkey, Qatar-official' (Reuters, 16 February 2018) <https://www.reuters.com/article/us-crypto-currency-venezuela/ venezuela-cryptocurrency-to-draw-investment-from-turkey-qatar-official-dUSKCN1G025S?utm _ campaign=trueAnthem\%3A+Trending+Content\&utm_content=5a87c16104d301339b95133f\&utm_ medium=trueAnthem\&utm_source=facebook $>$ accessed 24 January 2019.

28 Charlotte Krol, 'Bill Gates: Bitcoin is 'exciting' because it is cheap' (Telegraph, 3 October 2014) <http:// www.telegraph.co.uk/technology/11138905/Bill-Gates-Bitcoin-is-excitingbecause-it-is-cheap.html> accessed 21 February 2019.

29 Homeland Security (n 20); Angela Irwin and George Milad, 'The Use of Crypto-Currencies in Funding Violent Jihad' (2016) 19(4) Journal of Money Laundering Control 407: These articles demonstrate the use of cryptocurrency such as Bitcoin in the financing and funding of terrorist organisations and terrorist acts.

30 Europol, 'Internet Organised Crime Threat Assessment' European Cybercrime Centre, 24.

31 Carlisle (n 4) 27. 
web..$^{32}$ Marian also demonstrates how cryptocurrency is a potential super tax haven for the evasion of taxes. ${ }^{33}$ Terrorist organisations such as the Islamic State are also getting up to pace with the exploitation of cryptocurrency for terrorist activities. ${ }^{34}$ As Lagarde further notes, " $[T]$ he fact that the anonymity, the lack of transparency and the way in which cryptocurrency conceals and protects money laundering and financing of terrorism and all sorts of dark trades is just not acceptable'. ${ }^{35}$

Criminal activities with the use of cryptocurrency is not alien in Ghana. There is a high incidence of cybercrime such as credit card fraud in Ghana. ${ }^{36}$ The modus operandi of such cybercriminals is to obtain the credit card details of a person purchased on hidden online market sites on the dark web with cryptocurrency as the medium of exchange. The credit card details are used to procure services, and goods such as vehicles, branded clothing and electronic gadgets, among others, which are transported to Ghana and sold at knocked off prices.

Bitcoin, and significantly more sinister altcoins designed to offer far greater anonymity such as Darkcoin, Monero, Dash and Zcash, is gaining popularity as the currency of choice for cybercriminals. ${ }^{37}$ Bitcoin however, remains the virtual currency of choice for dark web activity. ${ }^{38}$ At this juncture, it is imperative to be wary of the inference that cryptocurrency offers absolute pseudonymity to users. As Bunjaku argues, cryptocurrency transactions are completely anonymous and at the same time fully transparent. ${ }^{39}$ This statement however, is not completely accurate. Reid and Harrigan have warned that

32 Online market sites such as Alphabay, Silk Road and Hansa. Access to such sites requires the use of an anonymiser. An anonymiser is a specialised software that provides access to blocked and hidden sites by altering and masking the IP address of an information technology (IT) device with a series of other IP addresses connected to the network. The Onion Router (TOR) is an example of an anonymiser: Homeland Security (n 20).

33 Omri Marian, 'Are Cryptocurrencies Super Tax Havens?' (2013) 112 Michigan Law Review.

34 Irwin and Milad (n 29) 407.

35 Christine Lagarde, 'An Even-handed Approach to Crypto-Assets' (IMFBlog, 16 April 2018) <https:// blogs.imf.org/2018/04/16/an-even-handed-approach-to-crypto-assets/> accessed 11 February 2019.

36 Ebenezer Sabutey, 'Ghana to get off PayPal's blacklist in 2019' (Joyonline, 10 May 2018) <https://www. myjoyonline.com/business/2018/May-10th/ghana-to-get-off-paypals-blacklist-in-2019.php> accessed 24 January 2019.

37 Europol (n 30) 11.

38 Steven Brown, 'Cryptocurrency and criminality: The Bitcoin Opportunity' (2016) 89(4) The Police Journal: Theory, Practice and Principles 327, 336.

39 Bunjaku and others (n 2). 
Bitcoin's touted anonymity is seriously flawed. ${ }^{40}$ They noted that, 'many organizations and services such as on-line stores that accept Bitcoin and exchanges have access to identifying information regarding their users, e.g., email addresses, shipping addresses, credit card and bank account details, Internet Protocol addresses, among others. If any of this information was publicly available, or accessible by law enforcement agencies, then the identities of users involved in related transactions may also be at risk'. ${ }^{11}$ Meiklejohn has also demonstrated how the real identities of Bitcoin users can be de-anonymised with the aid of clustering techniques. ${ }^{42}$

\section{Regulating the Faces of Cryptocurrency}

Cryptocurrency is intangible in nature, existing only in digital form. Lessig argues that regulation in the instance of technologies that are leveraged on the internet should consider who the user is, where the user is and the acts of the user on the internet. ${ }^{43}$ Hence this section considers the physical network participants that facilitate the use, holding and trading of cryptocurrency. This section identifies four main network participants namely; Miners, Users, Exchanges and Wallets.

\section{Miners}

Generally, cryptocurrency is created through the process of mining. Mining in the world of cryptocurrency refers to a dual process, that is, the generating of new cryptocurrency by solving complex codes and ciphers that add a block to the Blockchain, and the verification and validating of new transactions. The first process deals with the solving of complex codes and ciphers. Miners who solve such complex codes and ciphers add a block to the Blockchain, and are issued with a specified number of newly created cryptocurrency units, known as a block reward. The second is the verification and validation process. This process functions in a similar manner to a bank ensuring a customer has sufficient funds before honouring a demand by the customer. Hence miners solve the double spending problem in cryptocurrency by verifying and validating cryptocurrency transactions. A verified and validated transaction is recorded into a block of records on the Blockchain. Miners are then rewarded with a transactional fee for performing this task. The block reward and transactional fees serve as an incentive for 40 Guadamuz and Marsden (n 24) 12.

41 Fergal Reid and Martin Harrigan, 'An Analysis of Anonymity in the Bitcoin System' in Yaniv Altshuler and others (eds), Security and Privacy in Social Networks (Springer 2013) 197.

42 Sarah Micklejohn and others, 'A Fistful of Bitcoins: Characterizing Payments Among Men with No Names' (Proceedings of the 2013 Conference on Internet Measurement Conference, 2013).

43 Lawrence Lessig, Code: And Other Laws of Cyberspace, Version 2.0 (Basic books 2006) 23. 
miners to participate in the mining process.

Mining is a voluntary engagement initiated by the downloading of a specialised mining programme on a computer. The programme installed on the computer, with the aid of constant and relatively fast internet connection, mines on a computer. ${ }^{44}$ The complex codes and ciphers increases in difficulty as more blocks are added to the Blockchain. Thus mining on a basic personal computer has become impossible and inefficient for some cryptocurrency. Application Specific Integrated Circuits (ASIC) mining equipment have been developed to mine cryptocurrency more efficiently. Some miners also combine their computing power to mine in rigs and pools..$^{45}$ Ghana Dot Com, an IT solutions company based in Ghana, is attributed as the very first Bitcoin mining facility in Africa. ${ }^{46}$

\section{User}

A user refers to a person who obtains cryptocurrency as a medium of exchange, as a speculative asset ${ }^{47}$ or a hybrid of the two ${ }^{48}$ Cryptocurrency rely on cryptographical keys, that is a public key which corresponds to a private key, to encrypt the real identity and transactions of users. The public key is comparable to an email address, whilst the private key is similar to a password used to access an email account. The public key which is represented by a combination of alphanumeric characters identifies users. The public key does not reveal the personal identifiable information of a user. This therefore does not give out explicit information about the real owner of a unit of cryptocurrency. This feature makes users of cryptocurrency pseudonymous. The public key can also be represented by a Quick Response code (QR code).

\section{Exchanges}

Exchanges are entities that provide a market place for the trading and price discovery of cryptocurrency in circulation. Some exchanges also store users' cryptocurrency.

44 Paul Gil, 'A Beginner's Guide to Cryptocoin Mining' (Lifewire, 22 May 2018) <https://www.lifewire.com/ cryptocoin-mining-for-beginners-2483064> accessed 24 January 2019.

45 A mining rig is a specialised computer system used for mining of cryptocurrency. A mining pools also refers to the collaboration of miners who combine computing power to mine a block, and split the reward among the participants: Homeland Security (n 20) xiii.

46 'Bitcoin Mining in Ghana' <https://ice3x.co.za/bitcoin-mining-ghana/> accessed 24 January 2019.

47 In 2016, it was estimated that $54 \%$ of users of Coinbase, a popular digital currency exchange, obtained cryptocurrency strictly as an investment: Garrick Hileman and Michel Rauchs, 'Global Cryptocurrency Benchmarking Study' (Cambridge Centre for Alternative Finance 2017), 26.

48 Douglas King, 'Banking Bitcoin-Related Businesses: A Primer for Managing BSA/AML Risks' (2016) Retail Payments Risk Forum Working Paper, 3. 
Users can buy and sell cryptocurrency for fiat currency and, or other cryptocurrency at exchanges. Bitcoin is the most supported cryptocurrency on all exchanges. ${ }^{49}$ Users can also obtain cryptocurrency from a cryptocurrency Automated Teller Machines (ATM's). Cryptocurrency ATM's are specialised internet machines resembling ATM's developed to facilitate the trading and conversion of cryptocurrency to fiat currency. Most exchanges, that is an estimated $73 \%$ of exchanges, act as custodians of users' private keys thus have access to users funds. ${ }^{50}$ Access to the private key means control of the units of cryptocurrency in a wallet. This situation coupled with the surge in market prices of cryptocurrency has made exchanges popular targets for cybercriminals. ${ }^{51}$ Cybercriminals thus exploit security flaws in the computer systems of exchanges to obtain the private keys of users in order to appropriate units of cryptocurrency.

A quick google search of indigenous Ghanaian based exchange service providers will provide a list of exchange providers. Users can also trade with other users to obtain cryptocurrency on 'localbitcoins'. ${ }^{52}$

\section{Wallets}

A wallet is a software programme that securely stores, and facilitates the sending and receipt of cryptocurrency through the management of private and public cryptographical keys. ${ }^{53}$ Wallets provide an interface for users to track all previous transactions, determine their balance and ascertain the estimated transactional fees for transferring units of cryptocurrency. Majority of wallet service providers do not control the private keys of the users. ${ }^{54}$ Users have ultimate control of their accounts. Thus, users bare all the risks when it comes to ensuring the security of their private keys. Wallets can be downloaded on to mobile devices or computers. There seems to be no indigenous Ghanaian wallet service provider, although there is no evidence to suggest the nonexistence of one. A user can create multiple wallets with several or the same wallet service provider at the same time. Thus, estimating the exact number of Ghanaian users would prove quite impossible. The existence of other network participants such as indigenous Ghanaian miners and exchanges and the availability of wallet service providers online sufficiently 49 Hileman and Rauchs (n 47) 34.

50 Hileman and Rauchs (n 47) 37.

51 The first quarter of 2018 has been marked by the theft of about $\$ 700$ million worth of cryptocurrency in Japan and Italy alone: 'This Crypto Exchange Is Offering a \$250,000 Bounty for Hacker Tip-Offs' (Fortune, 12 March 2018) <http://fortune.com/2018/03/12/binance-cryptocurrency-hacker-tip-offs/> accessed 24 January 2019.

52 Localbitcoins <https://localbitcoins.com/country/GH> accessed 24 January 2019.

53 Hileman and Rauchs (n 47) 49.

$5473 \%$ of wallets do not control the private keys of users: ibid, 55 . 
demonstrates the existence of users in Ghana.

\section{To Regulate or Not to Regulate?}

The call for the regulation of cryptocurrency raises the question whether or not regulation is actually warranted. Some scholars have described cryptocurrency such as Bitcoin, as a bubble, a passing fad and a scam destined for the bins of history. ${ }^{55}$ This has raised comparisons of cryptocurrency with the tulip mania and the dotcom bubble. ${ }^{56}$ Notwithstanding, cryptocurrency is demonstrated to potentially transform the financial sector by providing cheap financial services to the underbanked and unbanked, and transforming the remittance industry by reducing transactional costs across borders. ${ }^{57}$ In the evolving continuum of cryptocurrency, states are also beginning to think more seriously about national forms of cryptocurrency which can result in the reduction of the high costs of printing and distributing banknotes, and the risks of counterfeits. ${ }^{58}$ Lagarde notes that, cryptocurrency rapid borderless, cheap and irreversible transfers can significantly transform financial activities in a meaningful and enduring way. ${ }^{59}$ Cryptocurrency is thus argued to offer tremendous opportunities for innovation and development, but it is also uniquely suited to facilitate illicit behaviour. ${ }^{60}$ Flowing from

55 Former Greek Finance Minister Varoufakis stated, 'There is a Bitcoin aristocracy, the Bitcoin early adopters, who accumulated very cheaply Bitcoins from the beginning. They have every reason to talk this thing up and lure people into like a Tulip-like mania or a pyramid, making extravagant claims [...] to (open and use a new Bitcoin ATM). This was all just hype': Evan Smart, 'Bitcoin Debate between Andreas Antonopoulos vs. The Greek Finance Minister' (Crytocoin News, 18 February 2015) <https://www.cryptocoinsnews.com/bitcoin-debate-andreasantonopoulos-vs-greek-finance-minister/> accessed 24 January 2019; Former US Federal Reserve Chairman Alan Greenspan, Nout Wellink, a former President of the Dutch Central Bank, and Nobel Laureate economist Robert Shiller, maintain that virtual currency is a passing fad or bubble, akin to Tulip mania in $17^{\text {th }}$ Century Netherlands. Nouriel Roubini, professor of economics at New York University, described Bitcoin was 'the mother of all bubbles' favoured by 'charlatans and swindlers': Angela Monaghan, 'Bitcoin biggest bubble in history, says economist who predicted 2008 crash' (The Guardian, 2 February 2018) <https://www.theguardian. com/technology/2018/feb/02/bitcoin-biggest-bubble-in-history-says-economist-who-predicted-2008crash> accessed 24 January 2019.

56 Lagarde (n 35); Lizzy McNeill and Sachin Croker, 'The truth about Tulip Mania' (BBC, 13 May 2018) < http://www.bbc.com/news/business-44067178 > accessed 24 January 2019; Jorn Madslien, 'Dotcom bubble burst: 10 years on' (BBC, 9 March 2010) <http://news.bbc.co.uk/2/hi/business/8558257.stm> accessed 24 January 2019.

57 Homeland Security (n 20) 163-165.

58 Misha Yang, 'Cryptocurrency in China: Light-Touch Regulation in Demand' (2016), $1<$ <ttps://papers. ssrn.com/sol3/papers.cfm?abstract_id=2792477> accessed 2 March 2019.

59 Lagarde (n 35).

60 Marian (n 3) 56. 
this, Marian notes that regulation aids cryptocurrency in achieving its positive potential whilst preventing cryptocurrency from becoming a vehicle for criminal activity. ${ }^{61}$

Drawing on the need to regulate, the Bank for International Settlements has identified five approaches to regulating cryptocurrency. ${ }^{62}$ First, regulators could decide to use moral suasions towards users and investors in order to highlight the relevant risks and to influence the market. The second approach borders on the regulation of specific entities such as those that enable interaction between digital currencies and traditional payment instruments. The third, posits that states can interpret existing regulations to apply to cryptocurrency and their network participants. The fourth approach supports the drafting of broader regulation similar to the regulations that applies to traditional payment methods, and last, the banning of the use cryptocurrency. Troeller builds on the approach of broadening the interpretation of existing laws to encompass cryptocurrency. ${ }^{63} \mathrm{He}$ notes that with this approach, 'it is still within a judge's discretion to include or not include cryptocurrency in the statutory scheme'. ${ }^{4}$ This could result in conflicting views limiting the certainty of the legal status of cryptocurrency.

Lim suggests that self-regulatory frameworks, where industry participants operate on agreed norms alongside guidance from regulators, may be more effective than heavyhanded regulatory approaches for regulating cryptocurrency. ${ }^{65}$ This suggestion of selfregulation is defined by Guadamuz and Marsden as the process where, 'governments provide support for mechanisms whereby users of virtual currencies can agree upon and enforce their own "community standards" and rules of conduct'. ${ }^{66}$ Carlisle toes to this line of argument by noting that when countries find that regulating network participants prove difficult, state agencies may encourage self-governing anti-money laundering approaches rather than formal regulation of network participants. This could include encouraging the cryptocurrency industry to develop voluntarily whilst incorporating meaningful standards that encourage responsible anti-money laundering practices. ${ }^{67}$

\section{Regulating Cryptocurrency in Ghana}

The Governor of the BoG in a speech acknowledged that, "[T]he future of money and

61 ibid, 59.

62 Committee on Payments and Market Infrastructures, 'Digital Currencies' (2015) Bank for International Settlements, 12.

63 Lauren Troeller, 'Bitcoin and Money Laundering (2016) 36 Review of Banking and Financial Law 159.

64 ibid, 166.

$65 \operatorname{Lim}($ n 5$) 22$.

66 Guadamuz and Marsden (n 24) 22.

67 Carlisle (n 4) 32. 
finance will be marked by innovation, new technologies and increased competition across borders'. ${ }^{68}$ The governor also cited the 2018 World Bank's Global Findex Report which marked Ghana's progress in financial inclusiveness as an improvement from 40.5 percent in 2014 to 58 percent in 2018 , driven mainly by digital financial services. ${ }^{69}$ This upward movement suggests an increase in the use of financial technologies, in spite of the digital divide in Ghana. ${ }^{70}$ The demonstrated nexus between unlawful activity and cryptocurrency also necessitates the need for regulation to pre-emptively protect the Ghanaian financial system from the potential mainstream use of cryptocurrency for unlawful activity. This section considers the possible existence of an existing regulatory framework regulating cryptocurrency in Ghana.

\section{Regulation as a Payment System}

On the $22^{\text {nd }}$ of January, 2018 , the BoG wary of the unlicensed nature of cryptocurrency under the repealed Payment Systems Act, 2003 (Act 622), issued a notice stating that, ' $[T]$ he public is therefore strongly encouraged to do business with only institutions licensed by the Bank of Ghana'. ${ }^{71}$ The Notice stated that the BoG has indicated its intention to regulate digital currencies with the drafting of the Payment Systems and Services Bill. ${ }^{72}$ This ambiguous indication did not specify which form of digital currencies the BoG seeks to regulate. ${ }^{73}$ The Director of Communications of the Bank of Ghana is quoted stating that the Payment Systems and Services Bill is expected to provide a regulatory framework for digital currencies. ${ }^{74}$ The Payment Systems and Services Bill has been passed into law.

68 Ernest Addison, Official Launch of Payswitch Company Limited (Payswitch conference, Accra 16

May 2018 <http://bog.gov.gh/privatecontent/Speeches/SPEECH\%20DELIVERED\%20BY\%20DR.\%20 ERNEST\%20ADDISON,\%20GOVERNOR,\%20AT\%20LAUNCH\%20OF\%2OPAYSWITCH\%20 COMPANY\%20LTD.pdf> accessed 19 January 2019.

69 ibid.

70 It is estimated that about $35 \%$ of the population representing about has access and is using the internet in Ghana: Kweku Zurek, 'Over 10 million Ghanaians use the internet - Report' (Graphic online, 19 February 2018) < https://www.graphic.com.gh/news/general-news/over-10-million-ghanaians-usingthe-internet-report.html> accessed 24 January 2019.

71 Bank of Ghana, 'Digital and Virtual Currencies Operations in Ghana' NOTICE NO. BG/GOV/ $\mathrm{SEC} / 2018 / 02$ [hereinafter referred to as the Notice].

72 Anita Arthur, 'BoG unfazed by digital currency inroads in Ghana' (Citifmonline, 3 February 2018) $<$ http://citifmonline.com/2018/02/03/bog-unfazed-digital-currency-inroads-ghana/> accessed 24 January 2019.

73 Digital currency can mean a digital representation of either virtual currency (non-fiat) or e-money (fiat) and thus is often used interchangeably with the term 'virtual currency': FATF Definition (n 13) 4.

74 The Director of Communications stated that, "We have a new Payment Systems \& Services Bill currently in parliament and once that is concluded, I'm sure the way forward will be made clear. It is something that the Bank of Ghana is taking very seriously and if you go through the release that we sent 
The Payment Systems and Services Act amends and consolidates the laws relating to payment systems, payment services and regulates institutions which carry on payment service and electronic money business and to provide for related matters. ${ }^{75}$

In the PSS Act, electronic money is defined as, 'monetary value which is stored electronically or magnetically, and represented by a claim on the issuer which is issued on receipt of funds, redeemable against cash and may be accepted by a person'. ${ }^{76}$ Electronic money is thus money which may be in the form of accepted legal tender of Ghana or the accepted legal tender of another country. Cryptocurrency such as Bitcoin and Ethereum is not recognised as legal tender in Ghana nor elsewhere. The definition of electronic money in the PSS Act is similar in substance to the definition of electronic money in the Electronic Money Directive of the European Union. ${ }^{77}$ The directive defines electronic money also as, 'electronically, including magnetically, stored monetary value as represented by a claim on the issuer which is issued on receipt of funds for the purpose of making payment transactions and which is accepted by a natural or legal person other than the electronic money issuer'. The European Court of Justice in distinguishing between cryptocurrency and electronic money held that, '[V]irtual currencies such as bitcoin, does not have a single issuer and instead is created directly in a network by a special algorithm'. ${ }^{78}$ The court further opined that Virtual currencies differ from electronic money, as defined in Directive 2009/110/EC ...in so far as, unlike that money, for virtual currencies the funds are not expressed in traditional accounting units, such as in euro, but in virtual accounting units, such as the 'bitcoin'. ${ }^{79}$ In analysing the decision of the court, it can be noted that cryptocurrency, as already stated, is issued through the process of mining and not on the receipt of funds. Also, cryptocurrency is not 'stored monetary value' as it is a unique medium of exchange and not accepted legal tender. ${ }^{80}$

The decisions of such foreign courts only have persuasive effects in the Ghanaian courts.

warning the public against doing transactions with Bitcoin , it is clearly stated there that it is something that the Bank has devoted enormous resources to.' He further stated that, 'We are also working with other international financial organizations to make sure that indeed the trend as we are witnessing now globally, if it's being adapted locally, we do have the resources to make sure that they are indeed properly regulated,': Arthur (n 72).

75 Long title of the Payment Systems and Services Act, 2019 (Act 987) [hereinafter know as PSS Act].

76 PSS Act, s 102.

77 Electronic Money Directive of the European Union, Directive 2009/110/EC.

78 Skatteverket v David Hedqvist Case C-264/14, 6 (July 2015), para 11.

79 ibid, para 12.

80 Samuel Alesu-Dordzi, 'Digital and Virtual currencies in Ghana: Did the Bank of Ghana miss the road?' (2018) Ghana Law Hub <https://ghanalawhub.com/digital-and-virtual-currencies-in-ghana-did-thebank-of-ghana-miss-the-road/> accessed 2 March 2019. 
As Sowah JA (as he then was) in the case Pokua v. State Insurance Corporation held, It is of course correct that our courts are not bound to follow decisions of foreign courts and are free to chart their own course; and so be it; but where the foreign piece of legislation is in pari materia with a similar enactment in our laws and where the words used are similar, it does seem prudent to have regard to the experience of those who have chartered the same course before and to observe in what manner we are in agreement with them or otherwise; and it is in this spirit that our courts should examine foreign decisions bearing ... I do not consider that English words must necessarily alter their meaning simply because the countries using the language might have different social and economic circumstances. ${ }^{81}$

\section{Regulation under Anti-Money Laundering Law}

Money laundering is the act of transferring illegally obtained money through legitimate people or accounts so that its original source cannot be traced. ${ }^{82}$ The origin of the term lies in the use of laundries and other cash businesses by organised crime to integrate proceeds of their crimes into the legitimate economy. ${ }^{83}$ It is estimated that $\$ 800$ billion $\$ 2$ trillion is laundered globally. ${ }^{84}$ Sadly, law enforcers and regulatory authorities seize only about one percent of money laundered internationally. ${ }^{85}$

Generally, the process of money laundering occurs in three stages, the placement, layering and integration stage ${ }^{86}$ Ellinger describes these three the stages as,

The first stage is the placement stage, where the proceeds of the crime are placed into the financial system. Moving on to the second stage, that is the layering stage, where proceeds are then moved usually through a series of transactions perhaps involving different entities, assets and jurisdictions in order to sever any audit trail hence tracing their origins harder. In the final stage, integration stage, the criminal resumes control of the proceeds free from any link to their criminal source. ${ }^{87}$

81 Pokua v. State Insurance [1973] 1 Ghana Law Report 335, 386.

82 Bryan Garner (ed in chief), Black's Law Dictionary (9th edn, 2009).

83 Eliahu Ellinger and others, Ellinger's Modern Banking Law (5 $5^{\text {th }}$ edn, OUP 2011).

84 United Nations Office on Drugs and Crime, 'Money-Laundering and Globalization' <https://www.unodc. org/unodc/en/money-laundering/globalization.html> accessed 24 January 2019.

85 Homeland Security (n 20) 98.

86 Ellinger and others ( $\mathrm{n} 83) 92$.

87 ibid. 
It is reported that $£ 3-£ 4$ billion pounds sterling of proceeds of unlawful activity has been laundered through cryptocurrency in Europe alone. ${ }^{88}$ The laundering of cryptocurrency is initiated by first placing the proceeds of illicit sources in the cryptocurrency system through the purchase of cryptocurrency from an exchange or cryptocurrency ATM's. As launderers convert illegally acquired cash to Bitcoin, their identity become pseudonymous and thereby frustrating authorities' ability to track their activity. The tainted units of cryptocurrency are transferred to multiple wallets and layered through series of transactions such as the trading in other altcoins or the purchase of goods and services. The launderer then receives goods or services, or money from an exchange or cryptocurrency ATM's free from the taint of crime. Some exchanges impose identification requirements on some transactions frustrating the obfuscating of the link of illegal proceeds to the real identities of the launderer. ${ }^{89}$

\section{Ghana's Anti Money Laundering Regulatory Framework}

Ghana's anti-money laundering legislation is couched in the Anti-Money Laundering Act, 2008 (Act 749) and the Anti-Money Laundering (Amendment) Act, 2014 (Act 874). ${ }^{90}$ Ghana's AML Act provides a two-prong regulatory approach to combat the menace of money laundering. The first approach is the criminalisation of the act of money laundering and related offences, and the second is the prevention of the money laundering by placing obligations and requirements on accountable institutions to detect and report suspicious transactions that may form or are proceeds of unlawful activity.

\section{Criminalising Money Laundering}

In Ghana, the act of money laundering is a crime punishable by a fine of not more than five thousand penalty units ${ }^{91}$ or to a term of imprisonment, not less than twelve months and not more than ten years or to both. The AML Act criminalises the activity of money laundering by stating;

A person commits an offence of money laundering if the person knows or ought to have known that property is or forms part of the proceeds of unlawful activity ${ }^{92}$ and the 88 Shiroma Silva, 'Criminals hide 'billions' in crypto-cash - Europol' (BBC, 12 February 2018) <http:// www.bbc.com/news/technology-43025787> accessed 24 January 2019.

89 'Exchanges such as Coinbase and Kraken, cloud-based wallet providers, and some ATM operators generally require various levels of identity verification based on transaction velocity and size': King (n 48) 9 .

90 Hereinafter referred to as the AML Act.

91 A penalty unit is worth GHS12 (Ghana Cedi).

92 Unlawful activity is defined as conduct which constitutes a serious offence, financing of terrorism, financing of the proliferation of weapons of mass destruction or other transnational organised crime or 
person; converts, conceals, disguises or transfers the property; conceals or disguises the unlawful origin, disposition, movement or ownership of rights with respect to the property; or acquires, uses or takes possession of the property. ${ }^{93}$

The AML Act further defines property as, 'assets of any kind situated in this country or elsewhere, regardless of its value, whether corporeal or incorporeal, movable or immovable, tangible or intangible, among others'. ${ }^{94}$

The AML Act does not define incorporeal or intangible assets. The Ghanaian courts have also not been confronted with the task of interpreting incorporeal and intangible assets pursuant to the AML Act to include cryptocurrency. My attention is thus drawn to Black's Law Dictionary which defines intangible assets as 'any nonphysical asset or resource than can be amortized or converted to cash...' and the word incorporeal as, 'having a conceptual existence but no physical existence. ${ }^{95}$ In light of the definitions provided, the ambit of property per the AML Act is wide in scope to include cryptocurrency. Thus a person can be convicted of the crime of money laundering of property in the form of cryptocurrency which is or forms part of proceeds of unlawful activity.

Obligations and Requirements of Accountable Institutions

The second approach is the placing of obligations and requirements on accountable institutions ${ }^{96}$ to detect and report suspicious transactions which may be the proceeds of unlawful activity. The AML Act imposes an obligation on such accountable institutions to keep records of transactions and the identities of persons who performed the transactions with the accountable institution. ${ }^{97}$ Such accountable institutions are also mandated to file suspicious transaction reports within twenty-four hours of knowledge of suspicious attempts or completed transactions, which the institution suspects the transaction is proceeds sourced illegally. ${ }^{98}$ What suffices as a suspicious transaction is not defined in the Act. In the case of $R v$. Da Silva, the court held that, " $T$ ] he essential element in the word "suspect" ... is that the defendant must think that there is a possibility, which is more

contravention of a law regarding any of these matters which occurs in this country or elsewhere: AML Act, s 1(2) as amended.

93 AML Act, s 1 as amended.

94 AML Act, s 51 (emphasis mine).

95 Garner (n 82).

96 AML Act, first schedule as amended by s 21 , the first schedule provides for accountable institutions which includes banks.

97 AML Act, s 23 as amended by s 8 .

98 AML Act s 30 as amended by s 11 . 
than fanciful, that the relevant facts exist. A vague feeling of unease will not suffice'. ${ }^{99}$

This second approach presents a problem as the network participants that facilitate the use, holding and trading of cryptocurrency are not licensed nor obligated to detect and report proceeds of unlawful activity in Ghana. However, cryptocurrency interacts with the Ghanaian financial system when a user buys or sells cryptocurrency with the national currency or e-money. This can be done through the physical exchange of money, a bank deposit or transfer of e-money at an exchange or with another user. Thus the payment of cryptocurrency with a bank deposit or transfer of e-money creates a nexus between exchanges and financial institutions regulated under the PSS Act.

\section{Confiscation and Seizure of Proceeds of Unlawful activity}

The Economic and Organised Crime Office (EOCO), is a specialised state agency that is empowered to seize, confiscate and recover property that a court of competent jurisdiction has adjudged as forming part or is proceeds of crime. ${ }^{100}$ The confiscation of cryptocurrency that is or forms part of proceeds of crime presents EOCO with a series of questions; what is the subject of such a seizure order?, where is the subject of seizure? And, how can EOCO enforce a seizure order in relation to cryptocurrency? Cryptocurrency such as Bitcoin is a subjective valuable record on a Blockchain. ${ }^{101}$ The property right is represented by a record on the Blockchain. ${ }^{102}$ The subject of a seizure order is property in the form of an intangible or incorporeal valuable record. Cryptocurrency, as already noted, is stored in a wallet or exchange. Ownership rights in cryptocurrency is prima facie presumed to be vested in the knowledge of the private key of a wallet. ${ }^{103}$ In order to enforce a seizure order of cryptocurrency the state agency exercising the order must have knowledge of the private key of a wallet. As demonstrated custodial exchanges that store users' cryptocurrency, have access to the private key of users. Thus a seizure order

99 [2006] EWCA Crim 1654.

100 Economic and Organised Crime Office 2010 (Act 804), s 2(b); s 24; Proceeds of crimes is defined as any property derived from or obtained through the commission of a serious offence: $s 74$ [hereinafter refer to as EOCO Act].

101 Witold Srokosz and Tomasz Kopyscianski, 'Legal and Economic Analysis of the Cryptocurrencies Impact On the Financial System Stability' (2015) Journal of Teaching and Education 619, 620.

102 ibid.

103 Brenig and others argue that ownership is established by knowing the corresponding public key to a wallet. but this is just control in my humble view thus we should look at the so I am trying to say I control with the private key directly or indirectly and it is as other ones do not give me access to control it. Hence what I own is a record on the block chain that is worth a number of units of cryptocurrency: Christian Brenig and others, 'Economic Analysis of Cryptocurrency Backed Money Laundering' (Twenty-Third European Conference on Information Systems (ECIS), Münster, 2015), 7. 
would be effective if issued against a custodial exchange or wallet. An authorised officer of the EOCO may also by notice in writing request for information from an exchange to assist in an investigation of a user. ${ }^{104}$ An authorised officer of the EOCO, in accordance with due process, is also empowered to search ${ }^{105}$ for tainted property ${ }^{106}$ by entering any land or premises and conduct a search in respect of tainted property to enforce a seizure order. ${ }^{107}$ Such a search on any land or premises includes property on the land or premise. ${ }^{108}$

\section{Conclusion: The Way Forward}

Ghana's finance minister during the budgetary statement for the year 2018 posited the financial policy of the government aimed at positioning Ghana as a regional financial services hub. ${ }^{109}$ Among the policies to strengthen the financial sector in Ghana is the conduct of a comprehensive study on cryptocurrency and Blockchain technology to enable the country position itself against any adverse effect on the economy and to provide an appropriate regulatory environment. ${ }^{110}$ This suggests that there is the momentum for the regulation of cryptocurrency in Ghana.

At this juncture, this article has demonstrated that cryptocurrency is not electronic money as defined in the PSS Act. Also, the article has shone light in the gaps, of the AML Act in relation the laundering of cryptocurrency in Ghana. Moving forward, Carlisle argues that anti-money laundering regimes would be most effective at the point where cryptocurrency come into contact with 'real' money and the formal financial system. ${ }^{111} \mathrm{He}$ further notes that, placing exchanges under anti-money laundering regulation enables exchanges to act as a gatekeeper to the fiat money world and to identify illicit actors who may be transferring funds between legal tender and cryptocurrency. ${ }^{112}$ Varriale supporting this argument also recommends that legislation should focus on the gateways, or the points at which people take real currency and transform it into virtual currency. ${ }^{113}$ Flowing

104 EOCO Act, s 19.

105 EOCO Act, s 25(4)(b), A search may be under a search warrant or emergency warrant.

106 EOCO Act, s 74: tainted property is defined as property used in or in connection with the commission of a serious offence or derived, obtained or realized as a result of the commission of a serious offence.

107 EOCO Act, S 25 1(b).

108 EOCO Act, S 252 (c).

109 Ken Ofori-Atta, 'The Budget Statement and Economic Policy of the Government of Ghana for the year 2018', para 838 .

110 ibid, para 846.

111 Carlisle (n 4) 13.

112 ibid.

113 Gemma Varriale, 'Bitcoin: How to Regulate a Virtual Currency' (2013) 32(6) International Financial 
from these assertions, regulation would be most effective when targeted at exchanges. This article considers the regulation of the exchanges in the cryptocurrency ecosystem. There is however, the risk of drafting nugatory regulatory frameworks for cryptocurrency which can be bypassed by trading online with other international exchanges. The fear of heavy handed regulation stifling the technological innovation of cryptocurrency is also ever present.

Thus, I propose an even-handed approach to regulating cryptocurrency in Ghana. Restating Guadamuz and Marsden, 'governments should provide supportfor mechanisms whereby users of virtual currencies can agree upon and enforce their own "community standards" and rules of conduct'. ${ }^{114}$ Drawing from the restatement, an optimal solution to regulation is for the BoG to persuade exchanges to self-regulate and serve as gatekeepers to monitor and detect property that is or forms part of proceeds of unlawful activity. This even-handed approach solicits the BoG to compel exchanges to adopt a rule of conduct or soft norms to guide their activities. The BoG can exercise its leverage over exchanges whose services are facilitated by the banks and regulated payment systems in Ghana to ensure compliance. Furthermore, the BoG can organise stakeholder meetings for the network participants in the Ghanaian cryptocurrency ecosystem to draft standards to regulate their activities in Ghana. Self-regulatory measures should include anti-money laundering procedures to ensure cryptocurrency is not being laundered through the exchanges in Ghana. An exchange that fails to comply would suffer reputational damage and possible isolation by the Ghanaian regulated financial institution.

Law Review, 2.

114 Guadamuz and Marsden (n 24). 